\title{
FROM GENERALIZED CAUCHY-RIEMANN EQUATIONS TO LINEAR ALGEBRAS
}

JAMES A. WARD

In a previous paper [1] the author gave a definition of analytic function in linear associative algebras with an identity. With each such algebra there was associated a set of partial differential equations called generalized Cauchy-Riemann differential equations which serve as a criterion to determine whether a given function is analytic in that algebra. A simplification for the commutative case has been given by Wagner [2].

The purpose of this paper is to give sufficient conditions (Theorem 2) that a set of equations

$$
\sum_{i, j=1}^{n} d_{k i j} \frac{\partial y_{i}}{\partial x_{j}}=0 \quad\left(k=1,2, \cdots,\left(n^{2}-n\right)\right),
$$

where the $d_{k i j}$ are constants in a field $F$, determine a linear associative commutative algebra $A$ over $F$ for which equations (1) are the generalized Cauchy-Riemann differential equations. This will enable us to find solutions of such a set by means of power series in the algebra.

Let $\epsilon_{1}, \epsilon_{2}, \cdots, \epsilon_{n}$ be a proper basis for a linear associative commutative algebra $A$ with an identity over the field $F$. Multiplication will be defined by

$$
\epsilon_{i} \epsilon_{j}=\sum_{k=1}^{n} C_{i j k} \epsilon_{k} \quad(i, j=1,2, \cdots, n)
$$

where the $C_{i j k}$ are in $F$. Denote by $R_{i}$ the matrix $\left(C_{i s r}\right)$ where $r$ is the row index and $s$ the column index. If $\alpha=a_{1} \epsilon_{1}+a_{2} \epsilon_{2}+\cdots+a_{n} \epsilon_{n}$ is any element of $A$, then $\alpha \leftrightarrow a_{1} R_{1}+a_{2} R_{2}+\cdots+a_{n} R_{n}$ is an isomorphism known as the first regular representation of $A$ by matrices.

Let $U$ denote a system of functions $y_{i}\left(x_{1}, x_{2}, \cdots, x_{n}\right)$ of $n$ variables $x_{1}, x_{2}, \cdots, x_{n}$ of $F$, and let the $y_{i}$ be analytic in a simply-connected region $R$ of $n$-space. Then $\eta=\sum_{i=1}^{n} y_{i} \epsilon_{i}$ will be called a function over $A$ of the variable $\xi=\sum_{i=1}^{n} x_{i} \epsilon_{i}$.

Since the algebra is commutative we define with Wagner $[2$, p. 456] that $\eta=\sum_{i=1}^{n} y_{i} \epsilon_{i}$ be an analytic function of $\xi$ if the $y_{i}$ are in $U$ and the Jacobian matrix $\left(\partial y_{r} / \partial x_{s}\right)$ is in $A$, that is, if there exist functions $z_{1}, z_{2}, \cdots, z_{n}$ such that

Received by the editors April 15, 1952. 


$$
\left(\frac{\partial y_{r}}{\partial x_{8}}\right)=z_{1} R_{1}+z_{2} R_{2}+\cdots+z_{n} R_{n}
$$

This implies a set of homogeneous relations among the $\partial y_{r} / \partial x_{s}$ called the generalized Cauchy-Riemann differential equations of the algebra with basis $\epsilon_{1}, \epsilon_{2}, \cdots, \epsilon_{n}$.

LEMмA. If $A$ is a linear associative commutative algebra of order $n$ with an identity over $F$, then a necessary condition that $\eta=\sum_{i=1}^{n} y_{i} \epsilon_{i}$ be analytic is that the components of $\eta$ satisfy a linearly independent system of differential equations

$$
\sum_{i, j=1}^{n} d_{k i j} \frac{\partial y_{i}}{\partial x_{j}}=0 \quad\left(k=1,2, \cdots,\left(n^{2}-n\right)\right),
$$

where the $d_{k i j}$ are constants in $F$ depending only on the multiplication constants $C_{i k j}$ of the algebra, and such that

$$
\sum_{i=1}^{n} d_{k i i}=0 \quad\left(k=1,2, \cdots,\left(n^{2}-n\right)\right) .
$$

By the definition of derivative the matrix equation

$$
u_{1} R_{1}+u_{2} R_{2}+\cdots+u_{n} R_{n}=\left(\frac{\partial y_{r}}{\partial x_{s}}\right)
$$

gives $n^{2}$ equations in the $n$ unknowns, with coefficients among the set $C_{i j k}$. From any $n$ of the equations (6) for which the determinant is not zero, we can solve for the $u_{i}$, and substitution in the remaining equations leads to $n^{2}-n$ equations of the form (4). Under a change of basis

$$
\epsilon_{i}=\sum_{j=1}^{n} t_{i j}^{\prime} \epsilon_{j}^{\prime}, \quad t_{i j} \in F \quad(i=1,2, \cdots, n),
$$

we have $x_{j}^{\prime}=\sum_{i=1}^{n} t_{i j} x_{i}$ and $y_{j}^{\prime}=\sum_{i=1}^{n} t_{i j} y_{i}$. Therefore

$$
\sum_{i, j=1}^{n} d_{k i j} \frac{\partial y_{i}}{\partial x_{j}}=\sum_{u, v=1}^{n} d_{k u v}^{\prime} \frac{\partial y_{u}^{\prime}}{\partial x_{v}^{\prime}} .
$$

Let $D_{k}=\left(d_{k r s}\right)$ and $D_{k}^{\prime}=\left(d_{k r s}^{\prime}\right)$; then

$$
D_{k}^{\prime}=T^{-1} D_{k} T
$$

where $T=\left(t_{r s}\right)$. Since the trace of a matrix is invariant under similarity transformation, we see that $\sum_{i=1}^{n} d_{k i i}$ is invariant under change 
of basis of the algebra $A$.

Wagner [2, equations 3] has shown that if $\epsilon_{1}$ is the identity, the generalized Cauchy-Riemann differential equations may be written

$$
-\frac{\partial y_{r}}{\partial x_{s}}+\sum_{i=1}^{n} C_{i s r} \frac{\partial y_{i}}{\partial x_{1}}=0 \quad(r, s=1,2, \cdots, n) .
$$

Since $\epsilon_{1}$ is the identity, $C_{1 s r}=\delta_{s r}$ (Kronecker $\delta$ ). For $s=1$ we get $n$ equations

$$
-\frac{\partial y_{r}}{\partial x_{1}}+\sum_{i=1}^{n} C_{11 r} \frac{\partial y_{i}}{\partial x_{1}}=0 \quad(r=1,2, \cdots, n)
$$

which are identically zero. The $n^{2}-n$ remaining equations are in the form (4). If, in (9), $r=s \neq 1$ we get

$$
-\frac{\partial y_{s}}{\partial x_{s}}+\frac{\partial y_{1}}{\partial x_{1}}+\sum_{i=2}^{n} C_{i s s} \frac{\partial y_{i}}{\partial x_{1}}
$$

because $C_{1 s s}=1$, hence $\sum_{i=1}^{n} d_{k i i}=-1+1=0$. If $r \neq s, s \neq 1, C_{1 s r}=0$ and the equations reduce to

$$
-\frac{\partial y_{r}}{\partial x_{i}}+\sum_{i=2}^{n} C_{i e r} \frac{\partial y_{i}}{\partial x_{1}}=0
$$

so that $d_{k i i}=0$ for every $i$. Hence in every case we have

$$
\sum_{i=1}^{n} d_{k i i}=0
$$

and the lemma is proved.

ThEOREM 1. If $A_{i}=\left(a_{i s r}\right)(i=1,2, \cdots, n)$ is a set of $n$ by $n$ matrices over $F$ such that

$$
A_{i} A_{j}=A_{j} A_{i} \quad(i, j=1,2, \cdots, n)
$$

and if there is a puch that

$$
a_{i p r}=\delta_{i r} \quad(i, r=1,2, \cdots, n),
$$

then

$$
A_{i} A_{j}=\sum_{t=1}^{n} a_{i j t} A_{t} \quad(i, j=1,2, \cdots, n)
$$

and the $A_{i}$ form a basis for a linear, commutative, associative algebra of order $n$ over $F$ with an identity element. 
By (12) the element of $u$ th row and $p$ th column of $A_{i} A_{j}$ is

$$
\sum_{t=1}^{n} a_{i t u} a_{j p t}=\sum_{t=1}^{n} a_{i t u} \delta_{j t}=a_{i j u}
$$

By change of notation we obtain the element of the $u$ th row and the pth column of $A_{j} A_{i}$ to be $a_{j i u}$. Thus we have

$$
a_{i j u}=a_{j i u} \quad(i, j, u=1,2, \cdots, n) .
$$

Another form of (11) is

$$
\sum_{t=1}^{n} a_{i t u} a_{j v t}=\sum_{t=1}^{n} a_{j t u} a_{i v t}
$$

Thus we have

$$
\begin{aligned}
A_{i} A_{j}=\left(\sum_{t=1}^{n} a_{i t r} a_{j s t}\right) & =\left(\sum_{t=1}^{n} a_{j t r} a_{i s t}\right) \\
& =\left(\sum_{t=1}^{n} a_{j t r} a_{s i t}\right) \\
& =\left(\sum_{t=1}^{n} a_{s t r} a_{j i t}\right) \\
& =\left(\sum_{t=1}^{n} a_{t s r} a_{i j t}\right) \\
& =\sum_{t=1}^{n} a_{i j t} A_{t .} .
\end{aligned}
$$

From (12) we see that the $A_{i}$ are linearly independent with respect to $F$. Equations (12) and (14) together show that $A_{p}=I$. Hence the $A_{i}$ form a basis for an algebra $A$ whose constants of multiplication are $C_{i j k}=a_{i j k}$, so that $R_{i}=A_{i}$.

Let

$$
f_{k} \equiv \sum_{i, j=1}^{n} d_{k i j} \frac{\partial y_{i}}{\partial x_{j}} \quad\left(k=1,2, \cdots,\left(n^{2}-n\right)\right)
$$

such that $d_{k i j}$ are in a field $F$ and the $f_{k}$ are linearly independent with respect to $F$. If there is a $p$ such that it is possible to solve for each $\partial y_{i} / \partial x_{j}$ in the system

$$
\sum_{i, j-} d_{k i j} \frac{\partial y_{i}}{\partial x_{j}}=0 \quad\left(k=1,2, \cdots,\left(n^{2}-n\right)\right)
$$


as a linear function of $\partial y_{1} / \partial x_{p}, \partial y_{2} / \partial x_{p}, \cdots, \partial y_{n} / \partial x_{p}$, then (17) may be put in a matrix form

$$
\left(\frac{\partial y_{r}}{\partial x_{s}}\right)=\left(\sum_{t=1}^{n} a_{t s r} \frac{\partial y_{t}}{\partial x_{p}}\right)=\sum_{t=1}^{n}\left(a_{t s r}\right) \frac{\partial y_{t}}{\partial x_{p}}=\sum_{t=1}^{n} A_{t} \frac{\partial y_{t}}{\partial x_{p}}
$$

by adjunction of the $n$ identities

$$
\frac{\partial y_{i}}{\partial x_{p}}=\frac{\partial y_{i}}{\partial x_{p}} \quad(i=1,2, \cdots, n) .
$$

THEOREM 2. Suppose the system of differential equations (17) has the property that for some fixed integer $p$, it implies the set (18). Suppose further that the matrices $A_{i}=\left(a_{i s r}\right), i=1,2, \cdots, n$, are commutative and

$$
\sum_{i=1}^{n} d_{k i i}=0 \quad\left(k=1,2, \cdots,\left(n^{2}-n\right)\right) .
$$

Then there is a uniquely determined linear, commutative associative algebra $A$, over $F$, for which (17) is a set of generalized Cauchy-Riemann differential equations (in the sense of the lemma).

Since each equation of (18) which is not an identity is a linear combination of the set (17), it follows from (19) that $a_{t p r}=\delta_{r t}$. Hence by Theorem 1 the $A_{i}(i=1,2, \cdots, n)$ form a basis for an algebra $A$ with $A_{p}=I$ as the identity element. It is seen that (17) and hence (16) is a set of generalized Cauchy-Riemann differential equations for the algebra.

For $n=2$ we have the

Corollary. A necessary and sufficient condition that the linearly independent equations

$$
\sum_{i, j=1}^{2} d_{k i j} \frac{\partial y_{i}}{\partial x_{j}}=0
$$

determine an algebra A for which (20) is a set of generalized CauchyRiemann differential equations is that

$$
d_{k 11}+d_{k 22}=0 \quad(k=1,2) .
$$

The necessity of (21) follows from the lemma.

Since (21) holds and the matrix of the coefficients of (20) is of rank 2, (20) may be put into form (18). Therefore either $A_{1}=I$ and therefore is commutative with $A_{2}$ or $A_{2}=I$ and is commutative with $A_{1}$. Therefore the corollary follows from Theorem 2. 
Ketchum [3, pp. 646 and 653] proved that every solution $y_{1}, y_{2}, \cdots, y_{n}$ of the generalized Cauchy-Riemann differential equations for a commutative algebra may be expressed in the form

$$
\eta=\sum_{i=1}^{n} y_{i} \epsilon_{i}=\sum_{j=0}^{\infty} a_{j} \xi^{j}
$$

where $\xi=\sum_{i=1}^{n} x_{i} \epsilon_{i}$. Therefore if equations (1) determine an algebra, the solutions of the equations may be obtained by use of power series in the algebra.

\section{REFERENCES}

1. James A. Ward, $A$ theory of analytic functions in linear associative algebras, Duke Math. J. vol. 7 (1940) pp. 233-248.

2. Raphael D. Wagner, The generalized Laplace equations in a function theory for commutative algebras, Duke Math. J. vol. 15 (1948) pp. 455-461.

3. P. W. Ketchum, Analytic functions of hypercomplex variables, Trans. Amer. Math. Soc. vol. 30 (1928) pp. 641-667.

UNIVERSITY OF KENTUCKY 